\title{
3 Research Square

\section{Cisplatin, Docetaxel and Cetuximab (TPEx) as First- line Treatment for Patients With Recurrent or Metastatic Head and Neck Cancer: A Multicenter Real-World Study}

\section{Agustín Falco}

Alexander Fleming Institute: Instituto Alexander Fleming

Mariano Leiva

Alexander Fleming Institute: Instituto Alexander Fleming

Albano Blanco

Alexander Fleming Institute: Instituto Alexander Fleming

Guido Cefarelli

Alexander Fleming Institute: Instituto Alexander Fleming

Andrés Rodriguez

Alexander Fleming Institute: Instituto Alexander Fleming

Juan Melo

Hospital Italiano de Buenos Aires

Federico Cayol

Hospital Italiano de Buenos Aires

Manglio Rizzo

Hospital Universitario Austral

Alejandro Sola

Fundación Centro Oncológico de Integración Regional (COIR)

Hernán Rodríguez Montani

Hospital italiano de Rosario

Matías Chacón

Alexander Fleming Institute: Instituto Alexander Fleming

Diego Enrico ( $\square$ denrico@alexanderfleming.org )

Alexander Fleming Institute: Instituto Alexander Fleming https://orcid.org/0000-0003-4121-6855

Federico Waisberg

Alexander Fleming Institute: Instituto Alexander Fleming

Research article 
Keywords: Recurrent and/or metastatic head and neck cancer, TPEx schema, Cetuximab, Docetaxel, Cisplatin

Posted Date: October 26th, 2020

DOI: https://doi.org/10.21203/rs.3.rs-95656/v1

License: (c) (1) This work is licensed under a Creative Commons Attribution 4.0 International License. Read Full License 


\section{Abstract}

\section{BACKGROUND}

The association of platinum, taxanes, and cetuximab has proven to be an effective and safe strategy for head and neck cancer treatment. Here we present a multi-institutional real-world experience of the TPEx schema as first-line therapy in advanced squamous cell carcinoma of the head and neck (SCCHN).

\section{METHODS}

This retrospective multicenter cohort study included patients with histologically confirmed recurrent or metastatic SCCHN treated with first-line TPEx regimen at five medical centers in Argentina between January 1, 2017, and April 31, 2020. Chemotherapy consisted of four cycles of docetaxel, cisplatin, and cetuximab, followed by cetuximab maintenance. Clinical outcomes and toxicity profiles were evaluated.

\section{RESULTS}

Twenty-four patients were included. Median age at diagnosis was 58 years (range 36-77). The majority of patients (83.3\%) received at least four chemotherapy cycles in the initial part. In the included group, overall response rate was $62.5 \%$, and three patients achieved a complete response (12.5\%). The median time to response was 2.4 months $(95 \% \mathrm{Cl} 1.3-3.5)$. With a median follow-up of 12.7 months $(95 \% \mathrm{Cl} 8.8-$ 16.6), the median progression-free survival was 6.9 months ( $95 \% \mathrm{Cl}$ : $6.5-7.3)$, and the overall survival rate at 12 months was $82.4 \%$. Two-thirds of patients reported at least one treatment-related adverse event, and $25 \%$ presented grade $3-4$ toxicities.

\section{CONCLUSIONS}

TPEx was an adequately tolerated regimen in our population. Median progression-free survival and overall response rates were consistent with recent reports in clinical trials evaluating this treatment combination.

\section{Introduction}

Squamous cell carcinoma of the head and neck (SCCHN) represents $5 \%$ of all newly diagnosed cancers, leading to over 300,000 deaths per year ${ }^{1}$. Despite appropriate primary treatments, in about $50 \%$ to $60 \%$ of patients with stage III to IV disease, loco-regionally relapse is evidenced ${ }^{2}$. Given that a significant proportion of these cases are not suitable for surgery or radiotherapy, systemic treatments and best supportive care are the preferred therapeutic options.

Up to the early 2000 s, the median overall survival (OS) of patients with metastatic disease was only 6 month $s^{3,4}$. This poor prognosis has encouraged significant research efforts in the last 15 years for developing novel drugs. In this setting, targeted therapy against the epidermal growth factor receptor (EGFR) cetuximab has proved substantial efficacy for recurrent or metastatic (R/M) SCCHN treatment in 
combination with 5-fluorouracil and platinum-based chemotherapy (EXTREME regimen) ${ }^{3}$. More recently, a new strategy using the immune checkpoint inhibitor pembrolizumab alone, or in combination with 5fluorouracil and platinum, became an appropriate first-line treatment for R/M SCCHN patients ${ }^{5-7}$.

Under these circumstances, the EXTREME regimen still represents a recommended first-line treatment option in selected scenarios, such as cases with PDL-1-negative tumors or when immunotherapy is contraindicated. Noteworthy, this treatment regimen may represent an attractive approach for patients with disease progression after first-line immune checkpoint inhibitors given as monotherapy ${ }^{8}$.

Taxanes have maintained widespread clinical use, particularly in solid tumors, since their discovery in the early 1970 s and several clinical trials have shown its antineoplastic activity against $\operatorname{SCCHN}^{9-11}$.

The fluorouracil substitution by a taxane seeks to take advantage of the potential immunogenic and proapoptotic synergy between cetuximab and docetaxel or paclitaxel ${ }^{12,13}$. Cetuximab, platinum, and taxane-based schedules were associated with promising survival results and cytoreductive properties in clinical studies ${ }^{14-18}$. In this context, TPExtreme was the first large, phase 3 , randomized trial comparing the TPEx regimen (cetuximab, taxane, and platinum) with the EXTREME schema at first-line setting ${ }^{19}$. This trial demonstrated similar efficacy outcomes in 539 R/M HNSCC patients, showing a median OS of 14.5 and 13.4 months using TPEx and EXTREME regimen, respectively. Furthermore, the TPEx arm had a more favorable toxicity profile, leading to better compliance of the planned treatment ( $72 \%$ vs. $44 \%)$, with fewer dose interruptions (10 vs. $27 \%$ ).

Based on these considerations and given the scarce real-world studies including patients that underwent this schema, we retrospectively evaluated the efficacy and safety of the TPEx regimen as first-line therapy in patients with R/M SCCHN.

\section{Patients And Methods}

\subsection{Study population and treatment characteristics.}

This retrospective multicenter cohort study included patients between January 1, 2017, and April 31, 2020, with histologically confirmed diagnosis of R/M SCCHN, that received TPEx as first-line treatment at five medical centers in Argentina. Chemotherapy consisted of four cycles of docetaxel $75 \mathrm{mg} / \mathrm{m}^{2}$, cisplatin $75 \mathrm{mg} / \mathrm{m}^{2}$ every three weeks, and cetuximab $(400 \mathrm{mg} / \mathrm{m} 2$ on day 1 of cycle 1 , then $250 \mathrm{mg} / \mathrm{m} 2$ weekly), with systematic granulocyte colony-stimulating factor (GCSF) support at each cycle. Patients with controlled disease continued with weekly $250 \mathrm{mg} / \mathrm{m} 2$ or every-2-weeks cetuximab $500 \mathrm{mg} / \mathrm{m}^{2}$ maintenance until disease progression or unacceptable toxicity. Demographic and clinicopathological characteristics, including age, ECOG performance status, smoking status, alcohol consumption, primary site tumor, and previous treatments were collected from medical charts and entered in a predefined centralized database. Efficacy and safety information was also retrieved, and treatment strategies, responses, adverse events, and discontinuation were also documented. 
Disease progression and treatment response were collected from medical charts. Treatment response was assessed by the investigator using CT or MRI scans in accordance with Response Evaluation Criteria in Solid Tumors (RECIST version 1.1). Adverse events were graded according to the National Cancer Institute Common Terminology Criteria for Adverse Events (version 4.0).

\subsection{Statistical analysis}

Data was summarized by frequency and percentage for categorical variables and by median and range for continuous variables. Progression-free survival (PFS) and OS of TPEx as first-line treatment were calculated from the date of therapy initiation to first documented relapse or death due to any cause, respectively. Data was censored on the last follow-up if the patient was alive. The duration of response (DOR) was defined as the time from first complete response (CR) or partial response to progressive disease or death. Survival curves were performed using the Kaplan-Meier method, and differences between groups were calculated using the log-rank test. All statistical analyses were performed using SPSS software version 23.0 (SPSS, Inc., Chicago, IL, USA).

\section{Results}

\subsection{Patients characteristics}

In this multicenter retrospective study, 24 patients with R/M SCCHN were included from five Argentinian medical centers. All patients received first-line chemotherapy with TPEx. Median age at diagnosis was 58 years (range $36-77)$, males represented $62.5 \%$ of the sample $(n=15)$, and the majority had ECOG $0-1(22$, 91.7\%) (Table 1). Smoking history was reported in 13 patients (54.2\%), and around one-third of the cases reported alcohol consumption. Of note, only two patients $(8.3 \%)$ had a body mass index $(\mathrm{BMI})<18.5$.

Previous treatments included definitive concomitant chemoradiotherapy (33.3\%), surgery (20.8\%), surgery plus radiotherapy (12.5\%), chemoradiotherapy (20.8\%), and definitive radiotherapy alone (4.2\%). Approximately half of the population had previously received cisplatin $(n=13,54.2 \%)$, and only two cases $(8.3 \%)$ had metastatic disease at diagnosis. The most common reason for treatment discontinuation was disease progression (58.3\%), and only two patients (8.3\%) discontinued treatment prematurely due to unacceptable toxicity. Notably, most cases $(83.3 \%)$ received at least four chemotherapy cycles in the induction part.

\subsection{Efficacy}

A total of three patients achieved a complete response (12.5\%), and in half of the cases a partial response was documented (Table 2). Remarkably, most of the cases benefited from TPEx therapy since the overall response rate (ORR) and disease control rate (DCR) were 62.5 and $87.5 \%$, respectively. The median time to response was 2.4 months $(95 \% \mathrm{Cl} 1.3-3.5)$.

No statistical differences were observed in terms of ORR or DCR among patients with only locoregional recurrence prior TEPx initiation compared to the rest of the included population (ORR 50\% [7/14], DCR 
85.7\% [12/14], and ORR 80\% [8/10], DCR 90\% [9/10], respectively; $p=0.21$ and $p=1.0$ ).

After a median follow-up of 12.7 months (95\% Cl 8.8-16.6), 14 progression events occurred. Median PFS and DOR were 6.9 months (95\% $\mathrm{Cl} 6.5-7.3)$ (Fig. 1) and 5.1 months (95\% $\mathrm{Cl} 3.0-7.2)$, respectively (Fig. 2). As expected, patients with documented tumor response showed a better PFS compared to the population with disease stabilization or progression (8.5 months [ $95 \% \mathrm{Cl} 5.9-11.1$ ] and 4.5 months [95\% $\mathrm{Cl} 4.0-5.0$ ], respectively; $p=0.034$ ) (Fig. 3). Notably, in the two out of three cases with documented CR a substantially longer PFS (22.3 and 18.8 months) and DOR (16.6 and 16.9) were observed. The OS rate at 12 months was $82.4 \%$. Remarkably, among the 14 patients that experienced disease progression with TPEx, 13 received second-line treatment with immunotherapy (pembrolizumab [n=9] and nivolumab [n=4]).

\subsection{Safety and adverse events}

Two-third of the patients reported at least one treatment-related adverse event and $25 \%$ at least one grade 3-4 adverse event. A summary of the safety profile is listed in table 3 . The most commonly reported hematological adverse events were febrile neutropenia (12.5\%), anemia (12.5\%), and hyponatremia/hypokalemia (12.5\%). Among non-hematological events, acne-like rash was the most frequent (33.3\%) related adverse event. Grade 3-4 nausea-vomiting, asthenia, and renal failure were noted in $4.2 \%$ of the cases. Only one patient experienced a grade 1 hypersensitivity reaction during taxane infusion.

Overall, serious adverse events were reported in five patients (20.8\%). Three of them developed febrile neutropenia, one acute renal failure, and the remaining patient was hospitalized due to grade 3 vomiting that required intravenous hydration. All cases continued treatment after resolving the toxicity. The median duration of hospitalization among patients with severe adverse events was six days (range 2-22). Additionally, no fatal events were reported. Globally, TPEx was associated with a low rate of adverse events leading to treatment interruption (12.5\%), discontinuation (8.3\%), or dose reduction (8.3\%).

\section{Discussion}

Despite substantial advances in the last decade, R/M SCCHN remains a significant clinical issue, given its associated high mortality rate. In this context, tumor response rate is an important goal in these patients given its association with symptoms improvement and better quality of life.

Over the past years, the EXTREME regimen became a preferred first-line strategy in R/M SCCHN patients ${ }^{3}$. While significant improvement in OS, PFS, and ORR was demonstrated in the cetuximab plus platinumfluorouracil arm of the pivotal phase 3 trial, $82 \%$ of the included patients experienced grade $3-4$ adverse events, mostly related to 5 -fluorouracil continuous infusion. Of note, all these findings were observed in fit patients, hence treatment decisions in this setting should be analyzed on a case by case basis. Clinical comorbidities, performance status, nutritional assessments, access to infusion pumps, or even availability for patient hospitalization are some of the considerations made in clinical practice before treatment definition. 
Given that not all patients can tolerate the EXTREME regimen, alternative treatment protocols were developed, mostly replacing 5-fluorouracil by taxanes. The phase 2 GORTEC study evaluated fifty-four patients with R/M SCCHN using cisplatin, docetaxel, and cetuximab in the first-line setting ${ }^{14}$. Median OS, PFS, and ORR were 14 months, 6.2 months, and 44.4\%, respectively. In this selected population, only 12 patients (22.2\%) experienced grade 4 adverse events. In another phase 2 trial, Bossi et al. randomized 201 patients with R/M SCCHN to first-line cetuximab plus cisplatin with or without paclitaxel ${ }^{15}$. Authors reported a median PFS of 7 months and an ORR of $51.7 \%$ in the cetuximab, cisplatin, and paclitaxel arm. With this regimen, $72.5 \%$ and $33 \%$ of the included patients presented grade $\geq 3$ and 4 adverse events, respectively.

Guigay and collaborators have recently published the results of a phase 3 trials that compared TPEx with EXTREME as first-line treatment in 539 patients $^{19}$. Overall survival, PFS and ORR were 14.5 vs. 13.4 months, 6.0 vs. 6.1 months, and 46 vs. $40 \%$, respectively, not finding significant differences between both arms. TPEx regimen was associated with $30 \%$ grade 4 adverse events, which was significantly lower than the $43 \%$ incidence reported with the EXTREME schema. Furthermore, an exploratory analysis of this trial showed a better quality of life in patients who received TPEx, mainly in global health status, physical functioning, role functioning, and scores of appetite ${ }^{20}$.

Remarkably, real-world data in this setting is scarce. Before the GORTEC trial, Guigay and collaborators presented the results of 30 patients treated with TPEx at Gustave Roussy Institute between 2011 and $2013^{21}$. In this group of patients, median PFS and OS were 6.0 and 13.6 months, respectively. A total of eight grade 3-4 adverse events were documented, including vomiting, mucositis, skin rash, diarrhea, hypersensitivity, and neutropenia. Additionally, Fuchs et al. reported similar results in a retrospective single-institution study, including 38 R/M SCCHN patients treated with TPEx at the Medical University of Vienna $^{22}$. In this study, the median OS, PFS, and ORR were 10.8 months, 6.3 months, and 50\%, respectively.

To the best of our knowledge, our study represents the first multicenter real-world data, including SouthAmerican patients treated with the TPEx schema. Notably, PFS and ORR were consistent with the previous clinical trials. Intriguingly, two patients with complete responses were associated with longer PFS, which may support that depth of response could be studied as a prognostic factor in patients with R/M HNSCC.

In our experience, the TPEx regimen was adequately tolerated by most of the analyzed patients. The incidence of grade 3-4 adverse events was surprisingly lower than expected (25\%), but it should be noted that five patients had treatment-related hospitalizations. Fortunately, no fatal toxicities were experienced.

Our experience confirms that the substitution of 5-fluorouracil by docetaxel may be a reasonable treatment strategy for R/M SCCHN patients. TPEx was incorporated as a standard regimen in our centers, considering that this regimen is associated with a lower duration of treatment infusions, lower total number of cycles, and the recent reports of safety and quality of life outcomes. These particularities are 
essential in low- and middle-income countries with limited access to infusion pumps. Furthermore, the instauration of simplified regimens has become of extreme importance during the COVID-19 pandemic ${ }^{23}$.

Our results should be interpreted with caution considering study limitations. This real-world data study was conducted in five private care centers, which can impact the high proportion of patients with access to immunotherapy after disease progression (92.9\%). The low number of included patients and the study retrospective nature may also hamper the extrapolation of our results to Hispanic and Latino-American populations. Additionally, our follow-up is not long enough to adequately analyze OS in our sample.

Finally, it should also be highlighted that the landscape in R/M SCCHN is evolving. First-line treatment strategies currently include immunotherapy given alone or in combination with chemotherapy ${ }^{7}$. Nevertheless, TPEx represents an adequate alternative for patients with R/M HNSCC without PDL-1 expression or as a subsequent treatment after disease progression with immune checkpoint inhibitors given as a monotherapy. It should be emphasized that drugs combination regimens, such as TPEx, have proven to be associated with higher ORR, which is particularly beneficial in patients with high tumor burden.

\section{Conclusions}

TPEx was a well-tolerated regimen in our population. Clinical outcomes, such as PFS and ORR, were comparable to the recently reported clinical trial using the same treatment schema. This regimen may be considered an attractive therapeutic strategy due to its more simplified administration, the total number of chemotherapy cycles, treatment tolerability. In this context, quality of life, cost of hospitalizations, and adverse event management should be carefully analyzed before deciding the best therapeutic plan for patients with R/M SCCHN.

\section{Abbreviations}

R/M SCCHN: recurrent or metastatic squamous cell carcinoma of the head and neck; EGFR: epidermal growth factor receptor; PFS: Progression-free survival; OS: Overall survival; TPEx regimen: cetuximab, taxane, and platinum; Cl: Confidence interval; ECOG PS: Eastern Cooperative Oncology Group PerformanceStatus; HPV: Human papillomavirus; DOR: Duration of response; IQR: interquartile range, NS: not specified.

\section{Declarations}

\section{Acknowledgments}

Not applicable

\section{Funding}


No specific funding for this work

\section{Authors'contribution}

Study concept and design: AF; Data acquisition and quality control of data: AF, ML, AB, GC, DE, FW; Data analysis and interpretation: $A F, M L, A B, D E, F W ;$ Manuscript preparation and editing: All authors; Manuscript review: All authors; All authors have read and approved the manuscript.

\section{Funding}

Not applicable.

\section{Availability of data and materials}

The datasets used and analyzed in the current study are available from the corresponding author on reasonable request.

\section{Ethics approval and consent to participate}

This study was approved by the Institutional Review Board of Alexander Fleming Institute. The requirement for written informed consent was waived owing to the retrospective nature of the study

\section{Consent for publication}

Not applicable.

\section{Competing interests}

The authors declare that they have no competing interests.

\section{References}

1. Mehanna H, Paleri V, West CML, et al: Head and neck cancer-Part 1: Epidemiology, presentation, and prevention. BMJ 341:c4684, 2010

2. Vokes EE, Weichselbaum RR, Lippman SM, et al: Head and neck cancer. N Engl J Med 328:184-194, 1993

3. Vermorken JB, Mesia R, Rivera F, et al: Platinum-based chemotherapy plus cetuximab in head and neck cancer. N Engl J Med 359:1116-1127, 2008

4. Mendenhall WM, Werning JW, Pfister DG: Cancer of the Head and Neck. In: Devita, Hellman, and Rosenberg's cancer: principles \& practice of oncology. 10th edition. Wolters Kluwer Health, 2015

5. Ferris RL, Blumenschein G, Fayette J, et al: Nivolumab for Recurrent Squamous-Cell Carcinoma of the Head and Neck. N Engl J Med 375:1856-1867, 2016 
6. Cohen EEW, Soulières D, Le Tourneau C, et al: Pembrolizumab versus methotrexate, docetaxel, or cetuximab for recurrent or metastatic head-and-neck squamous cell carcinoma (KEYNOTE-040): a randomised, open-label, phase 3 study. Lancet Lond Engl 393:156-167, 2019

7. Burtness B, Harrington KJ, Greil R, et al: Pembrolizumab alone or with chemotherapy versus cetuximab with chemotherapy for recurrent or metastatic squamous cell carcinoma of the head and neck (KEYNOTE-048): a randomised, open-label, phase 3 study. Lancet Lond Engl 394:1915-1928, 2019

8. Guidi A, Codecà C, Ferrari D: Chemotherapy and immunotherapy for recurrent and metastatic head and neck cancer: a systematic review. Med Oncol Northwood Lond Engl 35:37, 2018

9. Golden EB, Formenti SC, Schiff PB: Taxanes as radiosensitizers. Anticancer Drugs 25:502-511, 2014

10. Mekhail TM, Markman M: Paclitaxel in cancer therapy. Expert Opin Pharmacother 3:755-766, 2002

11. Misiukiewicz K, Gupta V, Bakst R, et al: Taxanes in cancer of the head and neck. Anticancer Drugs 25:561-570, 2014

12. Morelli MP, Cascone T, Troiani T, et al: Sequence-dependent antiproliferative effects of cytotoxic drugs and epidermal growth factor receptor inhibitors. Ann Oncol Off J Eur Soc Med Oncol 16 Suppl 4:iv61-68, 2005

13. Rose WC, Wild R: Therapeutic synergy of oral taxane BMS-275183 and cetuximab versus human tumor xenografts. Clin Cancer Res Off J Am Assoc Cancer Res 10:7413-7417, 2004

14. Guigay J, Fayette J, Dillies AF, et al: Cetuximab, docetaxel, and cisplatin as first-line treatment in patients with recurrent or metastatic head and neck squamous cell carcinoma: a multicenter, phase II GORTEC study. Ann Oncol Off J Eur Soc Med Oncol 26:1941-1947, 2015

15. Bossi P, Miceli R, Locati LD, et al: A randomized, phase 2 study of cetuximab plus cisplatin with or without paclitaxel for the first-line treatment of patients with recurrent and/or metastatic squamous cell carcinoma of the head and neck. Ann Oncol Off J Eur Soc Med Oncol 28:2820-2826, 2017

16. Tahara M, Kiyota N, Yokota T, et al: Phase II trial of combination treatment with paclitaxel, carboplatin and cetuximab (PCE) as first-line treatment in patients with recurrent and/or metastatic squamous cell carcinoma of the head and neck (CSPOR-HNO2). Ann Oncol Off J Eur Soc Med Oncol 29:1004-1009, 2018

17. Friesland S, Tsakonas G, Kristensen C, et al: Randomised phase II study with cetuximab in combination with 5-FU and cisplatin or carboplatin versus cetuximab in combination with paclitaxel and carboplatin for treatment of patients with relapsed or metastatic squamous cell carcinoma of the head and neck (CETMET trial). J Clin Oncol 36:6032-6032, 2018

18. Adkins D, Ley J, Atiq O, et al: Multicenter phase II trial of carbo- or cis-platin, nanoparticle albumin bound (nab)-paclitaxel, and ceTUXimabas first line therapy for recurrent/metastatic HNSCC: "the CACTUX Trial". Multidisciplinary Head and Neck Cancers Symposium (Scottsdale, AZ [abstract LBA9], 2018

19. Guigay J, Fayette J, Mesia R, et al: TPExtreme randomized trial: TPEx versus Extreme regimen in 1st line recurrent/metastatic head and neck squamous cell carcinoma (R/M HNSCC). J Clin Oncol 
$37: 6002-6002,2019$

20. Guigay J, Fayette J, Mesia R, et al: TPExtreme randomized trial: Quality of Life (QoL) and survival according to second-line treatments in patients with recurrent/metastatic head and neck squamous cell carcinoma (R/M HNSCC). J Clin Oncol 38:6507-6507, 2020

21. Even C, Bobillot B, Mayache-Badis L, et al: 997P - Results of Tpex (Docetaxel, Cisplatin, Cetuximab) Regimen Use in First Line Patients with Recurrent/Metastatic Squamous Cell Carcinoma of the Head and Neck (R/M Scchn) in a Single Institution. Abstr Book 39th ESMO Congr ESMO 2014 Madr Spain 26 - 30 Sept 2014 25:iv344, 2014

22. Fuchs H, Pammer J, Minichsdorfer C, et al: Modified biweekly cisplatin, docetaxel plus cetuximab (TPEx) as first-line treatment for patients with recurrent/metastatic head and neck cancer. Med Oncol Northwood Lond Engl 35:32, 2018

23. Waisberg F, Enrico D, Angel M, et al: Cancer Treatment Adaptations in the COVID-19 Era. JCO Oncol Pract 16:305-307, 2020

\section{Tables}


Table 1. Clinicopathological characteristics

Characteristics

Total
Median age (range), years

Sex

Male

Female

ECOG at TPEx initiation

0-1

2

Smoking History

Never

Current or former

NS

Alcohol consumption

Occasional or Regular

No

NS

Primary tumor site

Larynx

Oropharynx

Oral cavity

Hypopharynx

Other

p16 status (oropharyngeal carcinoma)

Positive

Negative

NS
Number of

Patients (\%)

24

$58(36-77)$

15 (62.5)

9 (37.5)

22 (91.7)

$2(8.3)$

$6(25)$

13 (54.2)

$5(20.8)$

8 (33.3)

7 (29.2)

9 (37.5)

7 (29.2)

$6(25)$

5 (20.8)

1 (4.2)

5 (20.8)

4 (66.7)

0

2 (33.3) 


\begin{tabular}{|ll|}
\hline Previous treatment & \\
\hline Concomitant chemoradiotherapy only & $8(33.3)$ \\
\hline Surgery only & $5(20.8)$ \\
\hline Surgery + radiotherapy & $5(20.8)$ \\
\hline Radiotherapy only & $3(12.5)$ \\
\hline No & $1(4.2)$ \\
\hline Extent of disease at TPEx initiation & $2(8.3)$ \\
\hline Locoregional recurrence only & $14(58.3)$ \\
\hline Locoregional recurrence + distant metastatic & $5(20.8)$ \\
\hline Metastatic disease & $5(20.8)$ \\
\hline $\begin{array}{l}\text { Time from initial diagnosis to recurrence } \\
\text { (median, IQR), months }\end{array}$ & $16.2(5.4-37.5)$ \\
\hline Metastatic or unresectable disease at diagnosis & $11(45.8)$ \\
\hline $\begin{array}{l}\text { Abbreviations: NS, not specified; ECOG, Eastern Cooperative Oncology Group; HPV, human papilloma } \\
\text { virus; IQR, interquartile range. }\end{array}$ \\
\hline
\end{tabular}


Table 2. Summary of treatment response

TPEx

$(\mathrm{N}=24)$

Type of response - no. (\%)

Complete

$3(12.5)$

Partial

$12(50)$

Stable disease

$6(25)$

Progression

$1(4.2)$

Non-assessable

Objective response rate - $\%$ of patients $(95 \% \mathrm{Cl})^{\mathrm{a}}$

62.5

Disease-control rate - \% of patients $(95 \% \mathrm{Cl})^{\mathrm{b}}$

87.5

Time to response $-\mathrm{mo}^{\mathrm{c}}$

Median (95\% Cl)

Duration of response - mo ${ }^{d}$

Median $(95 \% \mathrm{Cl})$

a. Objective response was considered to be a confirmed complete or partial response, as assessed by investigator.

b. The disease-control rate was considered to be a confirmed complete response, a partial response, or stable disease, as assessed by investigator.

c. Time to response was calculated with the use of the Kaplan-Meier method from the date of TPEx initiation to the date of the first documentation of a partial or complete response.

d. Duration of response was calculated with the use of the Kaplan-Meier method from the date of the first documented response until the date of documented disease progression, death, or the last response assessment in the absence of disease progression. 
Table 3. Adverse events of any cause during TPEx treatment.

\section{Event n (\%)}

Any treatment-related adverse event ${ }^{\mathrm{a}}$

Hematological

Febrile neutropenia

Anemia

Hyponatremia and/or hypokalemia

Hypomagnesemia

Thrombocytopenia

Non-hematological

Acne-like rash

Nausea - Vomiting

Asthenia

Diarrhea

Renal failure

Hypersensitivity

Oral mucositis

Any serious adverse event ${ }^{\mathrm{b}}$

Treatment-related deaths

Event leading to interruption of any treatment component ${ }^{c}$

Chemotherapy

Cetuximab

Event leading to discontinuation of any treatment component ${ }^{c}$

Chemotherapy

Cetuximab

Event leading to dose reduction
TPEx

$(\mathrm{N}=24)$

Any Grade

Grade 3-4

$18(75)$

$6(25)$

$3(12.5)$

$3(12.5)$

3 (12.5) $\quad 0$

$3(12.5) \quad 2(8.3)$

2 (8.3)

1 (4.2)

1 (4.2)

0

8 (33.3)

0

4 (16.7)

1 (4.2)

4 (16.7)

1 (4.2)

2 (8.3)

0

1 (4.2)

1 (4.2)

1 (4.2) 0

$1(4.2) \quad 0$
0

3 (12.5)

2 (8.3)

1 (4.2)

2 (8.3)

2 (8.3)

0

2 (8.3) 
a. The investigators determined whether adverse events were related to the treatment.

b. Adverse events that leaded hospitalization.

c. This category includes patients who interrupted or discontinued cisplatin, docetaxel, or cetuximab because of an adverse event at any time and patients who interrupted or discontinued cetuximab maintenance for an adverse event after completing the cycles of chemotherapy. Events were attributed to the specific treatment by the investigator.

\section{Figures}

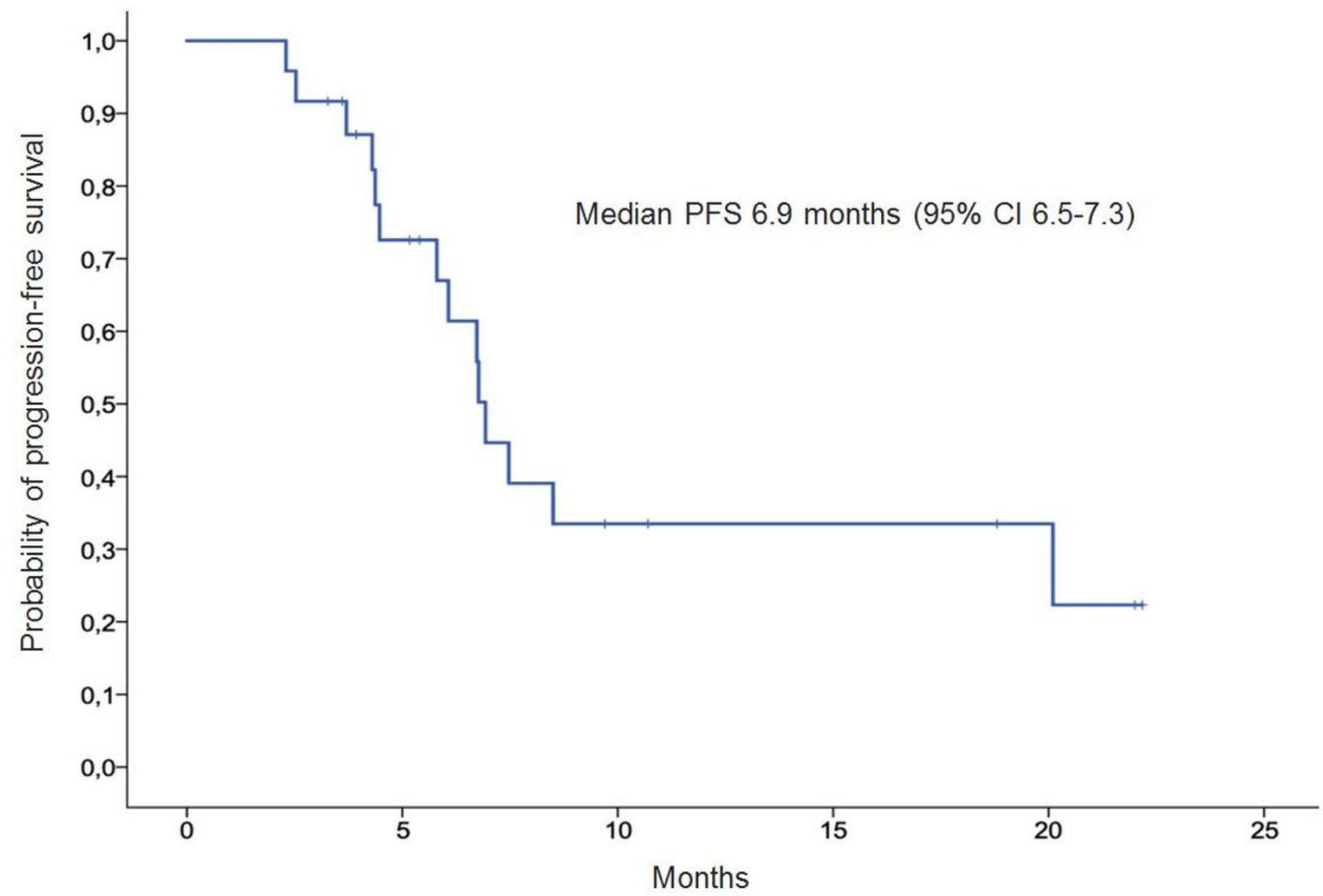

\section{Figure 1}

Kaplan-Meier curves for progression-free survival (PFS). 


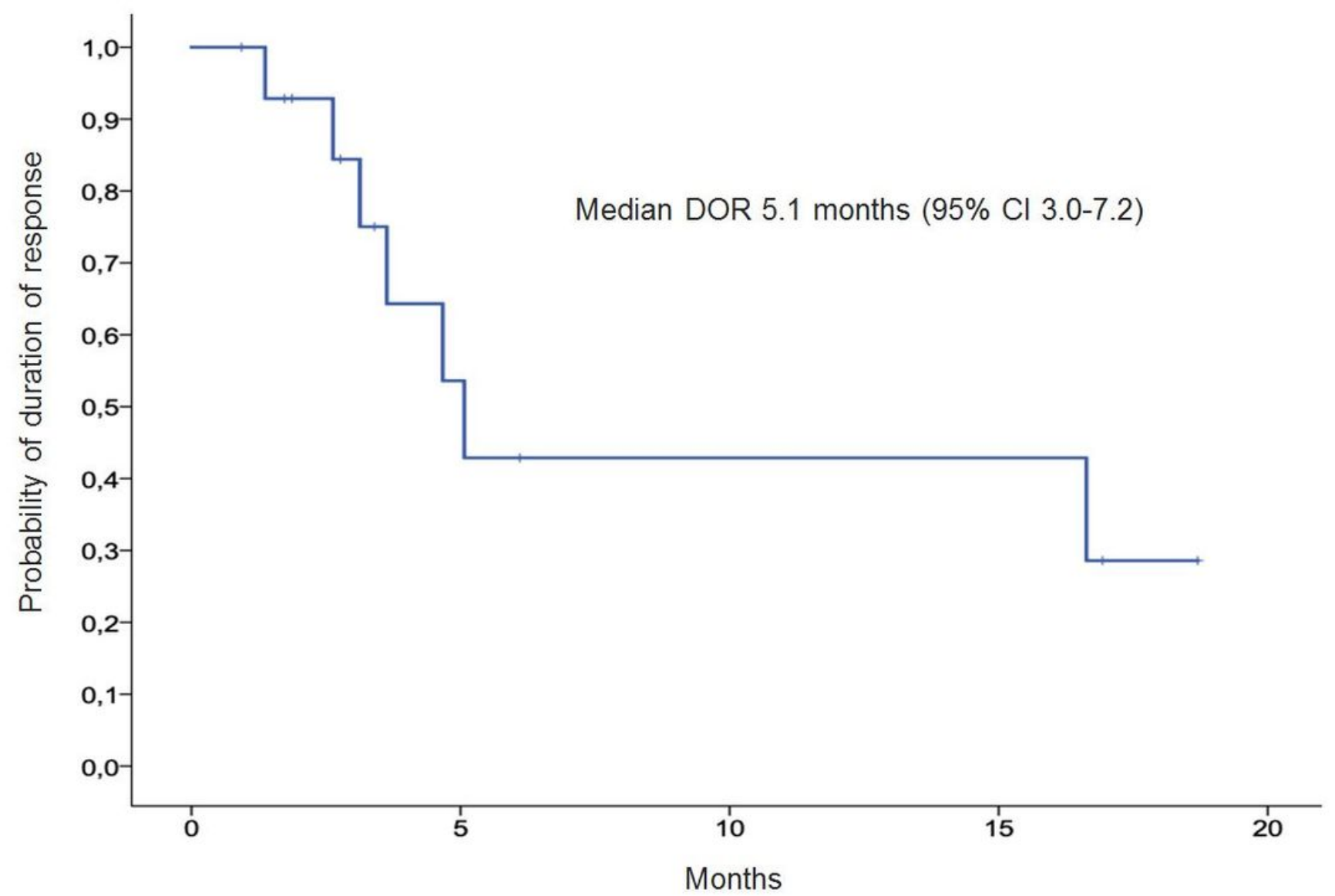

Figure 2

Kaplan-Meier curves for duration of response (DOR). 


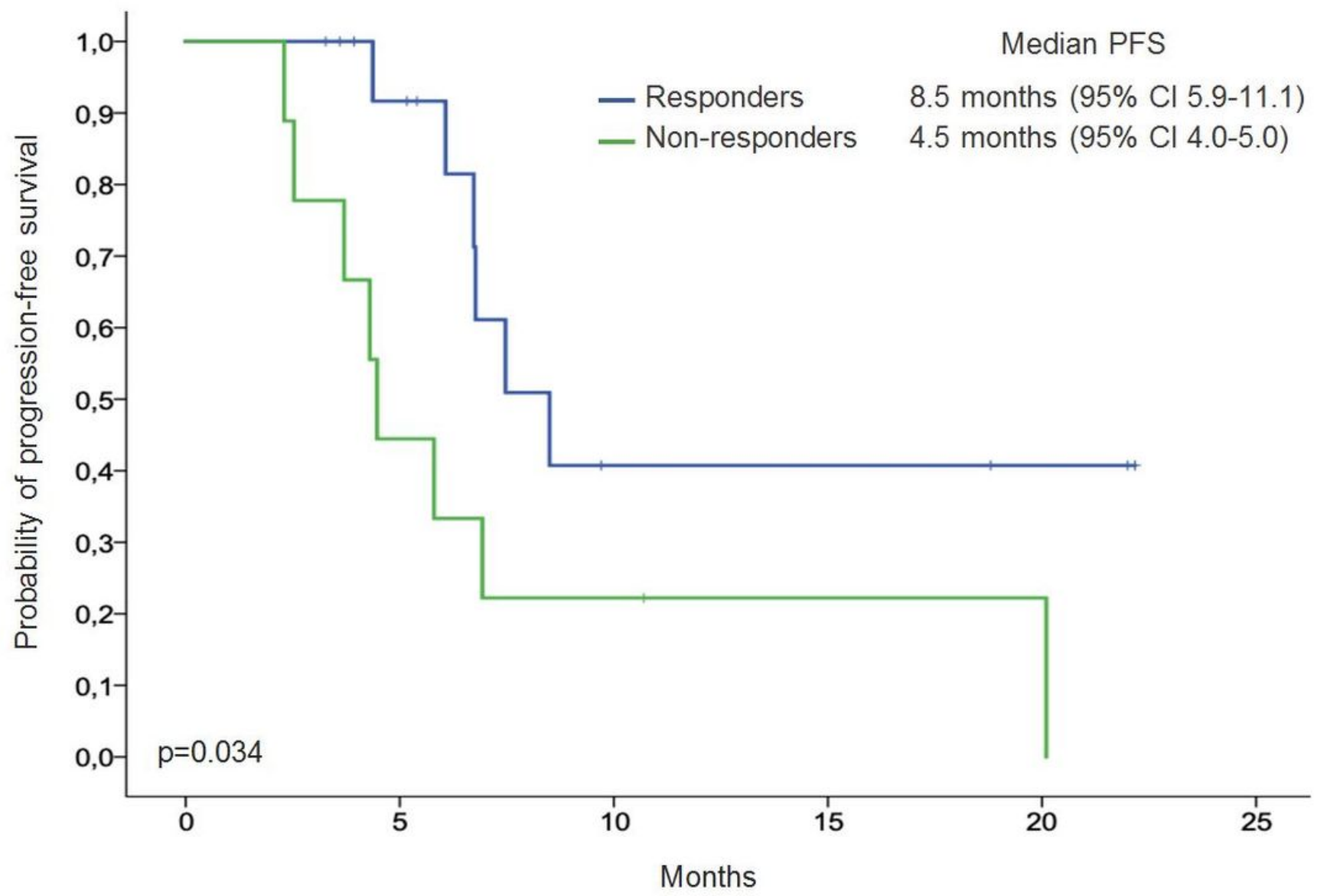

Figure 3

Kaplan-Meier curves for progression-free survival (PFS) according to response. 